\title{
Correlations and fluctuations measured by the CMS experiment in $\mathrm{pp}$ and $\mathrm{PbPb}$
}

\author{
Wei Li for the CMS collaboration \\ Massachusetts Institute of Technology, 77 Mass Ave, Cambridge, MA 02139-4307, USA \\ E-mail: davidlw@mit.edu
}

\begin{abstract}
Measurements of charged dihadron angular correlations are presented in proton-proton $(\mathrm{pp})$ and Lead-Lead $(\mathrm{PbPb})$ collisions, over a broad range of pseudorapidity and azimuthal angle, using the CMS detector at the LHC. In very high multiplicity pp events at center-of-mass energy of $7 \mathrm{TeV}$, a striking "ridge"-like structure emerges in the two-dimensional correlation function for particle pairs with intermediate $p_{\mathrm{T}}$ of $1-3 \mathrm{GeV} / c$, in the kinematic region $2.0<|\Delta \eta|<4.8$ and small $\Delta \phi$, which is similar to observations in heavy-ion collisions. Studies of this new effect as a function of particle transverse momentum are discussed. The long-range and shortrange dihadron correlations are also studied in $\mathrm{PbPb}$ collision at a nucleon-nucleon center-of-mass energy of $2.76 \mathrm{TeV}$, as a function of transverse momentum and collision centrality. A Fourier analysis of the long-range dihadron correlations is presented and discussed in the context of CMS measurements of higher order flow coefficients.
\end{abstract}

Measurements of dihadron azimuthal correlations [1, 2, 3, 4, 5, 6] have provided a powerful tool to study the properties of the strongly interacting medium created in ultrarelativistic nuclear collisions. At RHIC, dihadron azimuthal correlation measurements extending to large relative pseudorapidities resulted in the discovery of a ridge-shaped correlation in central $\mathrm{AuAu}$ collisions between particles with small relative azimuthal angles $(|\Delta \phi| \approx 0)$, out to very large relative pseudorapidities $(|\Delta \eta|)$ [2, 5]. Recently, a striking ridge structure has also been observed in very high multiplicity proton-proton (pp) collisions at a center-of-mass energy of $7 \mathrm{TeV}$ at the LHC by the Compact Muon Solenoid (CMS) Collaboration [7], posing new challenges to the understanding of these long-range correlations.

Dihadron correlations for charged particles have been measured extensively by the CMS experiment in pp collisions at $\sqrt{s}=7 \mathrm{TeV}$ and $\mathrm{PbPb}$ collisions at a nucleonnucleon center-of-mass energy $\left(\sqrt{s_{N N}}\right)$ of $2.76 \mathrm{TeV}$ over a large phase space. The nearly $4 \pi$ solid-angle acceptance of the CMS detector is ideally suited for studies of both shortand long-range particle correlations. A detailed description of the CMS detector can be found in Ref. [8]. The dihadron correlations analysis technique has been described in Ref. [9].

$\ddagger$ For the full list of CMS authors and acknowledgments, see appendix "Collaborations". 
Long-range, near-side $(\Delta \phi \approx 0)$ ridge-like azimuthal correlations for $2.0<|\Delta \eta|<$ 4.8 have recently been observed for the first time in high multiplicity pp collisions at $\sqrt{s}=7 \mathrm{TeV}$ [7]. The novel structure resembles similar features observed in relativistic heavy-ion experiments. This feature is most evident in the intermediate transverse momentum range of both $1<p_{\mathrm{T}}^{\text {trig }}<3 \mathrm{GeV} / c$ and $1<p_{\mathrm{T}}^{\text {assoc }}<3 \mathrm{GeV} / c$. Following up the first observation of the ridge correlation structure in high multiplicity pp collisions, new results are presented to study the detailed event multiplicity, transverse momentum and pseudorapidity gap $(\Delta \eta)$ dependence of the ridge effect using the full statistics data collected in 2010. With a dedicated high multiplicity tracking high-level trigger (HLT) setup, the CMS experiment has a unique capability in studying this effect.
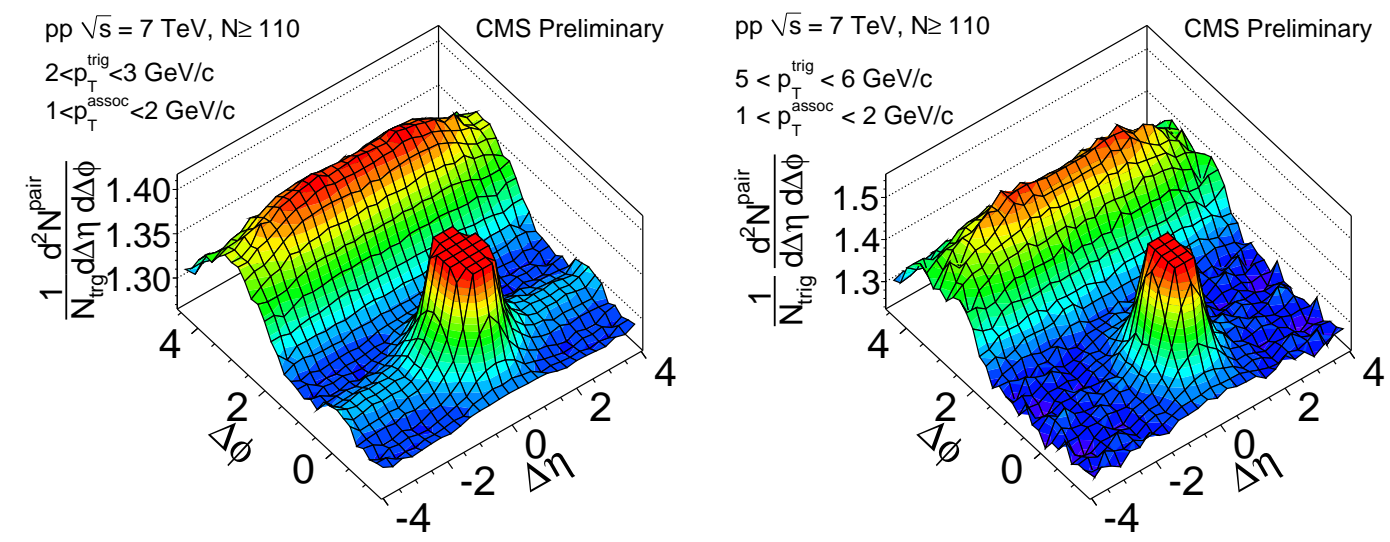

Figure 1: Two-dimensional (2-D) per-trigger-particle associated yield of charged hadrons as a function of $\Delta \eta$ and $\Delta \phi$ with jet peak cutoff for better demonstration of the ridge from high multiplicity $(N \geq 110)$ pp collisions at $\sqrt{s}=7 \mathrm{TeV}$, for $2<p_{\mathrm{T}}^{\text {trig }}<3 \mathrm{GeV} / \mathrm{c}$ and $1<p_{\mathrm{T}}^{\text {assoc }}<2 \mathrm{GeV} / \mathrm{c}$ (left), and $5<p_{\mathrm{T}}^{\text {trig }}<6 \mathrm{GeV} / \mathrm{c}$ and $1<p_{\mathrm{T}}^{\text {assoc }}<2 \mathrm{GeV} / \mathrm{c}$ (right).

The per-trigger-particle associated yield distribution of charged hadrons as a function of $\Delta \eta$ and $\Delta \phi$ in high multiplicity $(N \geq 110)$ pp collisions at $\sqrt{s}=$ $7 \mathrm{TeV}$ with trigger particles with $2<p_{\mathrm{T}}^{\text {trig }}<3 \mathrm{GeV} / c$ and associated particles with $1<p_{\mathrm{T}}^{\text {assoc }}<2 \mathrm{GeV} / \mathrm{c}$ is shown in Fig. 1 (left). The ridge-like structure is clearly visible at $\Delta \phi \approx 0$ extending to $|\Delta \eta|$ of at least 4 units as previously observed in Ref. [7]. However, at higher $p_{\mathrm{T}}^{\text {trig }}$ of $5-6 \mathrm{GeV} / c$ as presented in Fig. 1 (right), the ridge almost disappears.

In order to fully explore the detailed properties of both short-range jet-like correlations and long-range ridge-like structure, especially its dependence on event multiplicity and transverse momentum, the associated yield distributions are obtained in eight bins $(2 \leq N<35,35 \leq N<45,45 \leq N<60,60 \leq N<90, N \geq 90$, $N \geq 110, N \geq 130, N \geq 150)$ of charged particle multiplicity and six bins $(0.1-1$, $1-2,2-3,3-4,4-5$ and $5-6 \mathrm{GeV} / \mathrm{c})$ of particle transverse momentum. The 1-D $\Delta \phi$ azimuthal correlation functions are calculated by integrating over the $0.0<|\Delta \eta|<1.0$ and $2.0<|\Delta \eta|<4.0$ region, defined as the jet region and ridge region, respectively. 

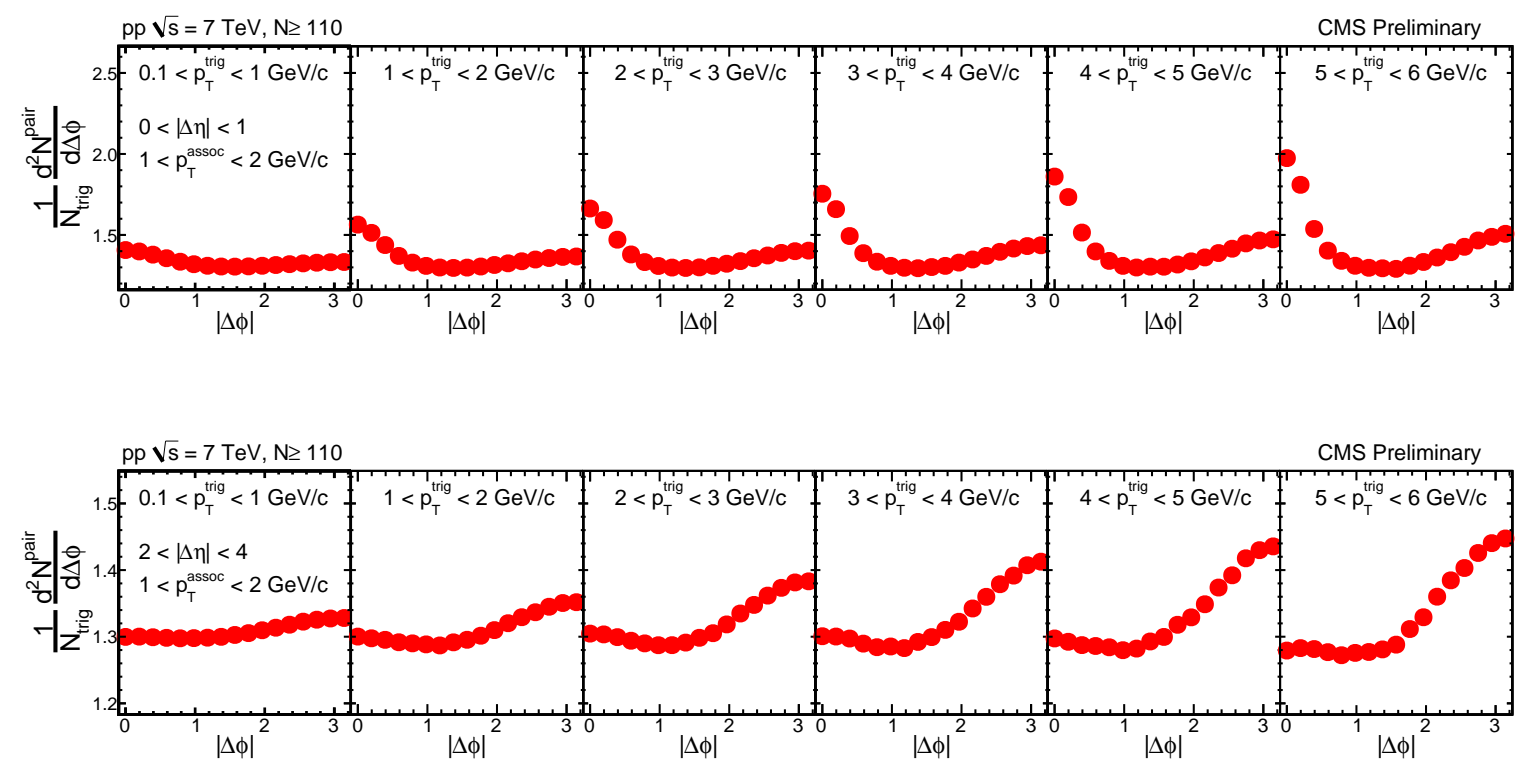

Figure 2: Short-range $(|\Delta \eta|<1)$ and long-range $(2<|\Delta \eta|<4)$ per-trigger-particle associated yields of charged hadrons as a function of $|\Delta \phi|$, from the high multiplicity $(N \geq 110)$ pp collisions at $\sqrt{s}=7 \mathrm{TeV}$, for $\operatorname{six} p_{\mathrm{T}}^{\text {trig }}$ and $p_{\mathrm{T}}^{\text {assoc }}$ bins. The error bars are statistical only and too small to be visible in most of the panels.

Fig. 2 shows the results in the jet and ridge region respectively, for $N \geq 110$ in six bins of $p_{\mathrm{T}}^{\text {trig }}$ at $1<p_{\mathrm{T}}^{\text {assoc }}<2 \mathrm{GeV} / c$.
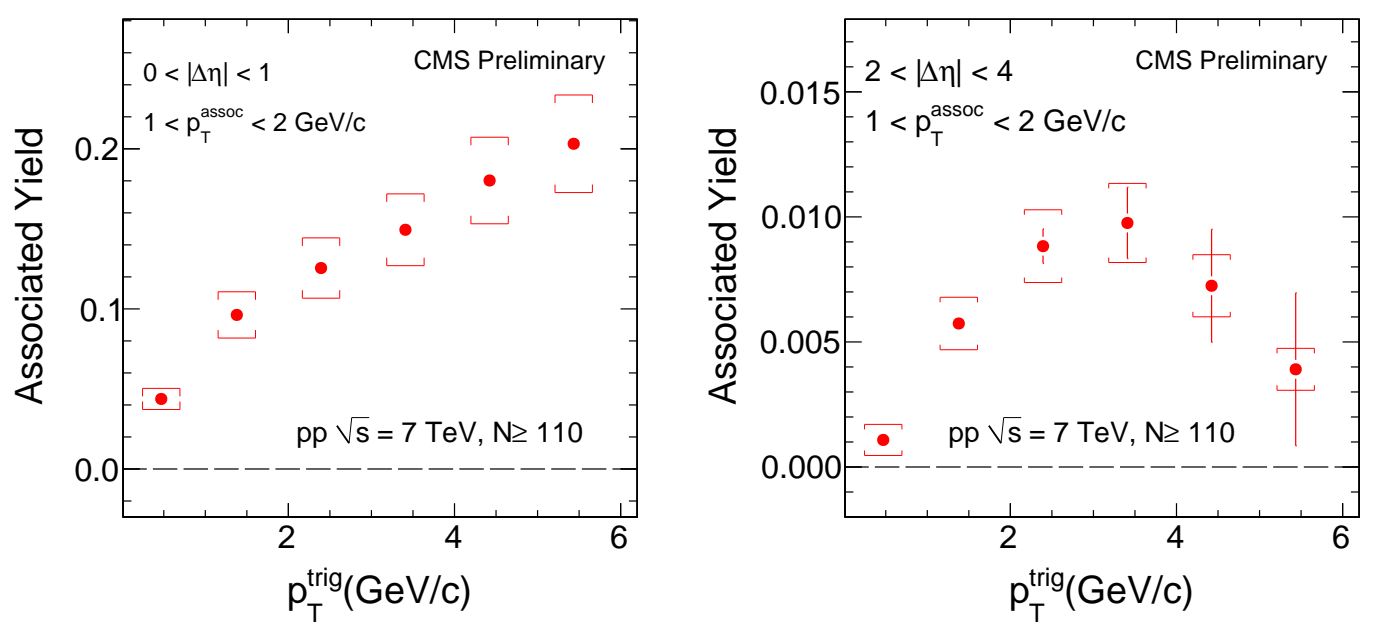

Figure 3: Integrated near-side associated yields for the short-range jet region $(0<$ $|\Delta \eta|<1)$ and the long-range ridge region $(2<|\Delta \eta|<4)$, with $1<p_{\mathrm{T}}^{\text {assoc }}<2 \mathrm{GeV} / c$, above the minimum level found by the ZYAM procedure, as a function of $p_{\mathrm{T}}^{\text {trig }}$ for $N \geq 110$ of pp collisions at $\sqrt{s}=7 \mathrm{TeV}$. The statistical uncertainties are shown as bars, while the brackets denote the systematic uncertainties. 
In the next step, the near-side (small $\Delta \phi$ region) integrated associated yield is calculated for both jet and ridge regions relative to the minimum of the distribution. The details of this so-call "ZYAM" procedure can be found in Ref. [9]. Figure 3 shows the integrated near-side associated yield for both the jet and ridge region correlations with $1<p_{\mathrm{T}}^{\text {assoc }}<2 \mathrm{GeV} / c$ (the $p_{\mathrm{T}}$ range where the ridge effect appears to be strongest) as a function of $p_{\mathrm{T}}^{\text {trig }}$ for $N \geq 110$. The jet yield increases with $p_{\mathrm{T}}^{\text {trig }}$ as expected due to the increasing contributions from high $p_{\mathrm{T}}$ jets. The ridge yield first increases with $p_{\mathrm{T}}^{\text {trig }}$, reaches a maximum around $p_{\mathrm{T}}^{\text {trig }} \sim 2-3 \mathrm{GeV} / c$ and drops at higher $p_{\mathrm{T}}^{\text {trig }}$.
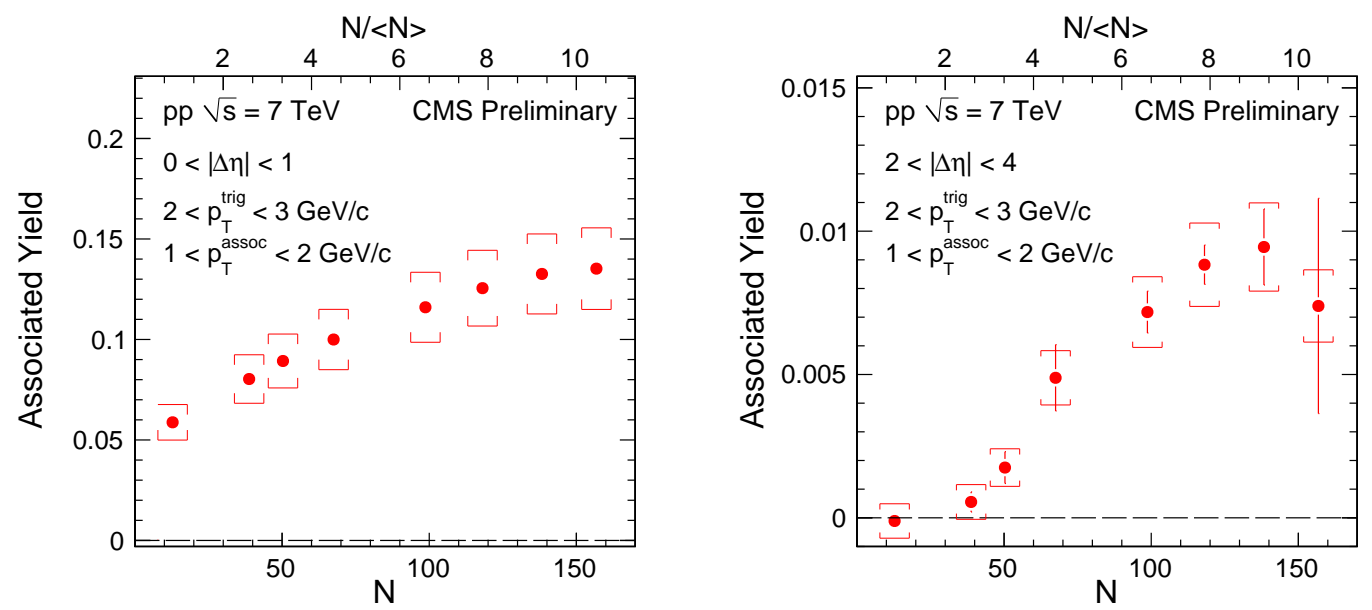

Figure 4: Integrated near-side associated yields for the short-range jet region $(0<$ $|\Delta \eta|<1)$ and the long-range ridge region $(2<|\Delta \eta|<4)$, with $2<p_{\mathrm{T}}^{\text {trig }}<3 \mathrm{GeV} / c$ and $1<p_{\mathrm{T}}^{\text {assoc }}<2 \mathrm{GeV} / c$, above the minimum level found by the ZYAM procedure, as a function of event multiplicity from pp collisions at $\sqrt{s}=7 \mathrm{TeV}$. The statistical uncertainties are shown as bars, while the brackets denote the systematic uncertainties.

The multiplicity dependence of the near-side associated yield in the jet and ridge region is illustrated in Fig. 4 for one transverse momentum bin of $2<p_{\mathrm{T}}^{\text {trig }}<3 \mathrm{GeV} / c$ and $1<p_{\mathrm{T}}^{\text {assoc }}<2 \mathrm{GeV} / c$, the $p_{\mathrm{T}}$ bin where the ridge effect appears to be strongest. The ridge effect gradually turns on with event multiplicity around $N \sim 50-60$ (about four times of the average multiplicity in minimum bias events) and smoothly increases toward high multiplicity region.

Moving onto $\mathrm{PbPb}$ collisions, dihadron correlations have been systematically studied as a function of centrality and particle transverse momentum. An example of the 2-D correlations for trigger particles with $4<p_{\mathrm{T}}^{\text {trig }}<6 \mathrm{GeV} / c$ and associated particles with $2<p_{\mathrm{T}}^{\text {assoc }}<4 \mathrm{GeV} / c$ is shown in Fig. 5, starting from $0-5 \%$ corresponding to the most central collisions in the upper left-hand corner, to the $70-80 \%$ bin corresponding to the most peripheral ones in the lower right-hand corner. In the $0-5 \%$ most central $\mathrm{PbPb}$ collisions, a clear and significant ridge-like structure is observed at $\Delta \phi \approx 0$, which extends all the way to the limit of the measurement of $|\Delta \eta|=4$. For more peripheral collisions, a $\cos (2 \Delta \phi)$ component becomes prominent, which is attributed to the elliptic 
flow effect.

The results of the 1-D $\Delta \phi$ correlations for the $0-5 \%$ most central $\mathrm{PbPb}$ collisions are shown in Fig. 6. The associated yield per trigger particle in the range of $2<$ $p_{\mathrm{T}}^{\text {assoc }}<4 \mathrm{GeV} / c$ is extracted for five different $p_{\mathrm{T}}^{\text {trig }}$ intervals $(2-4,4-6,6-8,8-10$, and 10$12 \mathrm{GeV} / \mathrm{c}$ ), and for both jet and ridge region. A comparison to PYTHIA $8 \mathrm{pp} \mathrm{MC}$ events at $\sqrt{s}=2.76 \mathrm{TeV}$ is also shown, with a constant added to match the $\mathrm{PbPb}$ results at $\Delta \phi=1$ in order to facilitate the comparison. In $\mathrm{PbPb}$, the height of the ridge structure decreases as $p_{\mathrm{T}}^{\text {trig }}$ increases and has largely vanished for $p_{\mathrm{T}}^{\text {trig }} \approx 10-12 \mathrm{GeV} / c$, a feature that is qualitatively similar to what is observed in high multiplicity pp collisions, as was presented earlier. The diminishing height of the ridge with increasing $p_{\mathrm{T}}^{\text {trig }}$ was not evident from previous measurements in $\mathrm{AuAu}$ collisions.
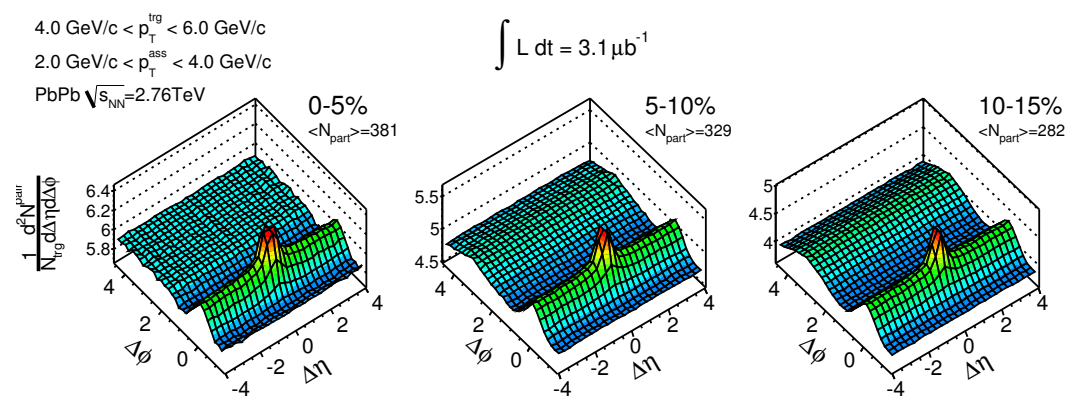

CMS Preliminary
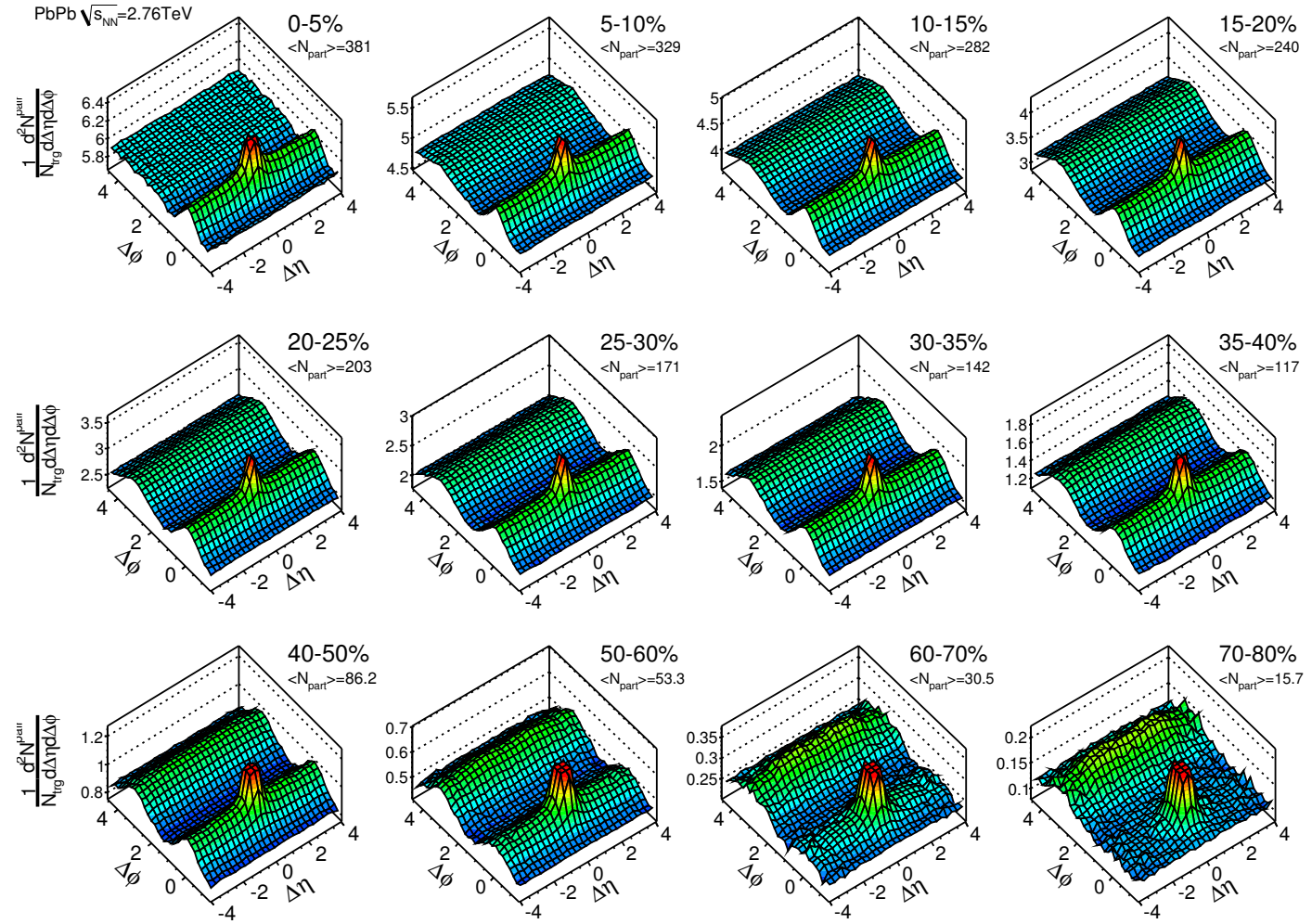

Figure 5: Two-dimensional (2-D) per-trigger-particle associated yield of charged hadrons as a function of $\Delta \eta$ and $\Delta \phi$ for $4<p_{\mathrm{T}}^{\text {trig }}<6 \mathrm{GeV} / c$ and $2<p_{\mathrm{T}}^{\text {assoc }}<4 \mathrm{GeV} / c$ in 12 centrality classes of $\mathrm{PbPb}$ collisions at $\sqrt{s_{N N}}=2.76 \mathrm{TeV}$. The centrality labeling is such that $0-5 \%$ is the most central five percent of $\mathrm{PbPb}$ collisions.

Motivated by the idea of understanding the long-range ridge effect in the context of higher-order hydrodynamic flow induced by the initial geometric fluctuations [10, 11, 12, 13, an approach of quantifying the observed long-range correlations using a Fourier decomposition technique is investigated. The 1-D $\Delta \phi$-projected distribution is decomposed into Fourier series using the following expression: 

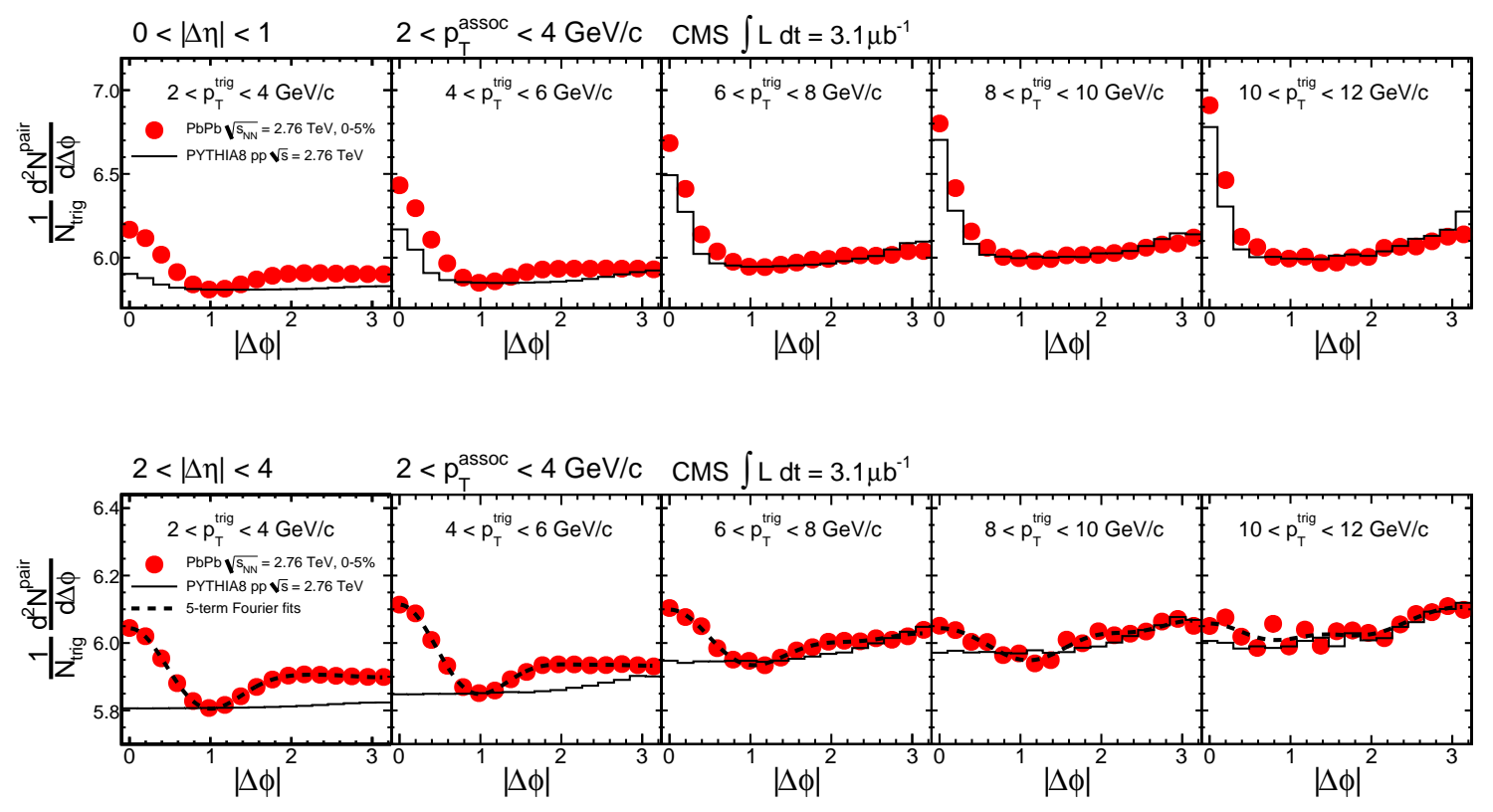

Figure 6: Short-range $(|\Delta \eta|<1)$ and long-range $(2<|\Delta \eta|<4)$ per-trigger-particle associated yields of charged hadrons as a function of $|\Delta \phi|$ from the $0-5 \%$ most central $\mathrm{PbPb}$ collisions at $\sqrt{s_{N N}}=2.76 \mathrm{TeV}$, requiring $2<p_{\mathrm{T}}^{\text {assoc }}<4 \mathrm{GeV} / c$, for five different intervals of $p_{\mathrm{T}}^{\text {trig }}$. The PYTHIA 8 pp MC results (solid histograms) are also shown, shifted up by a constant value to match the $\mathrm{PbPb}$ data at $\Delta \phi=1$ for ease of comparison. The error bars are statistical only and are too small to be visible in most of the panels. The systematic uncertainty of $7.6 \%$ for all data points is not shown in the plots.

$$
\frac{1}{N_{\text {trig }}} \frac{d N^{\text {pair }}}{d \Delta \phi}=\frac{N_{\text {assoc }}}{2 \pi}\left\{1+\sum_{n=1}^{\infty} 2 V_{n \Delta} \cos (n \Delta \phi)\right\},
$$

where $N_{\text {assoc }}$ represents the total number of dihadron pairs per trigger particle for a given $|\Delta \eta|$ range and $\left(p_{\mathrm{T}}^{\text {trig }}, p_{\mathrm{T}}^{\text {assoc }}\right)$ bin. The $1-\mathrm{D} \Delta \phi$ projections are fitted by the first five terms in the Fourier series, the resulting fits being shown as the dashed lines in Fig. 6. The data are well described by this fit. The extracted first five Fourier coefficients from the fit are presented in Figure 7 as functions of $p_{\mathrm{T}}^{\text {trig }}$ for $2<p_{\mathrm{T}}^{\text {assoc }}<4 \mathrm{GeV} / c$ for the $0-5 \%$ most central $\mathrm{PbPb}$ collisions and for the PYTHIA8 pp MC simulation. The PYTHIA8 results are scaled by the ratio of $N_{\text {assoc }}^{\text {PYTia8 }} / N_{\text {assoc }}^{\mathrm{PbPb}}$ in order to remove the trivial dilution of the correlation strength with increasing multiplicity.

If the observed correlation was purely driven by the single-particle azimuthal anisotropy arising from the hydrodynamic expansion of the medium [14], the extracted $V_{n \Delta}$ components would factorize into the flow coefficients $v_{n}$ (i.e., $v_{2}$ for anisotropic elliptic flow):

$$
V_{\mathrm{n} \Delta}\left(p_{\mathrm{T}}^{\text {trig }}, p_{\mathrm{T}}^{\text {assoc }}\right)=v_{\mathrm{n}}^{\mathrm{f}}\left(p_{\mathrm{T}}^{\text {trig }}\right) \times v_{\mathrm{n}}^{\mathrm{f}}\left(p_{\mathrm{T}}^{\text {assoc }}\right),
$$




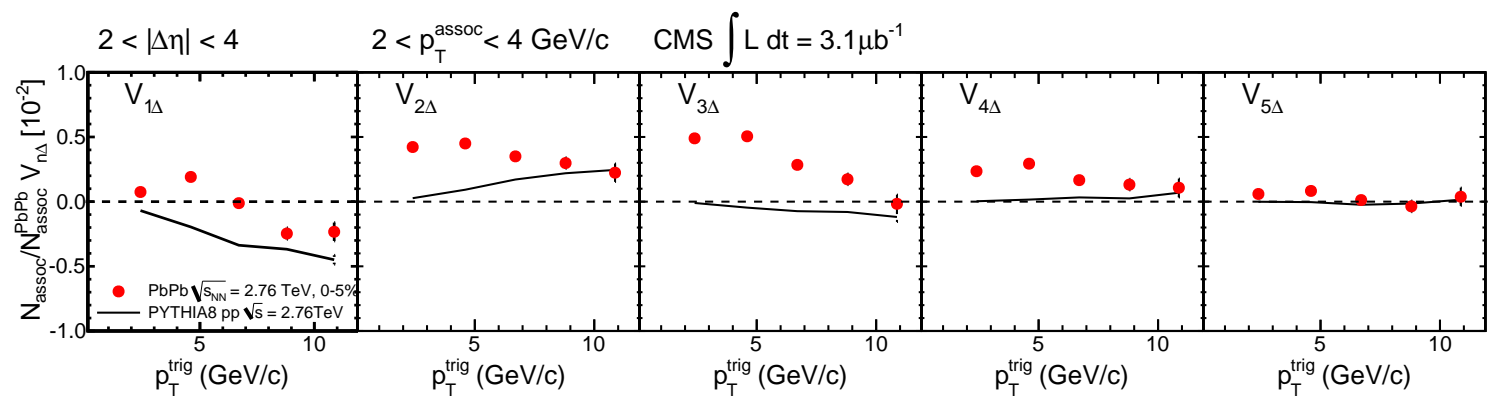

Figure 7: Fourier coefficients, $V_{1 \Delta}, V_{2 \Delta}, V_{3 \Delta}, V_{4 \Delta}$, and $V_{5 \Delta}$, extracted as functions of $p_{\mathrm{T}}^{\text {trig }}$ for $2<p_{\mathrm{T}}^{\text {assoc }}<4 \mathrm{GeV} / c$ for the $0-5 \%$ most central $\mathrm{PbPb}$ collisions at $\sqrt{s_{N N}}=$ $2.76 \mathrm{TeV}$. The error bars represent statistical uncertainties only. The solid lines show the predictions from the PYTHIA 8 simulation of pp collisions at $\sqrt{s}=2.76 \mathrm{TeV}$.

where $v_{\mathrm{n}}^{\mathrm{f}}\left(p_{\mathrm{T}}^{\text {trig }}\right)$ and $v_{\mathrm{n}}^{\mathrm{f}}\left(p_{\mathrm{T}}^{\text {assoc }}\right)$ are the flow coefficients for the trigger and associated particles [10].

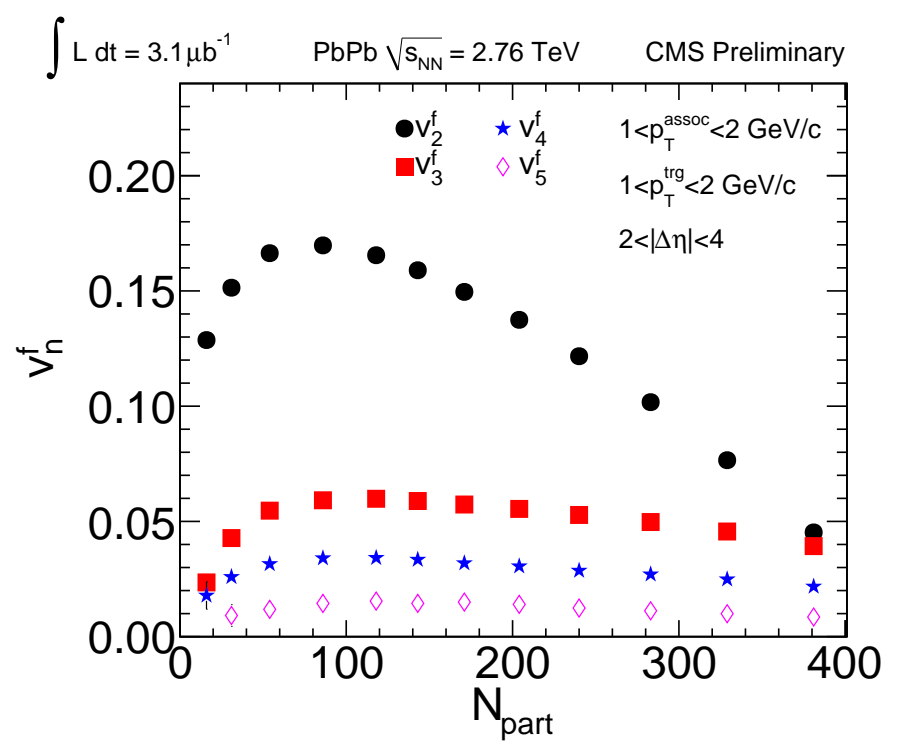

Figure 8: The flow harmonics $v_{2}^{\mathrm{f}}, v_{3}^{\mathrm{f}}, v_{4}^{\mathrm{f}}$ and $v_{5}^{\mathrm{f}}$ extracted from long-range $(2<|\Delta \eta|<4)$ azimuthal dihadron correlations for $1<p_{\mathrm{T}}^{\text {trig }}<2 \mathrm{GeV} / c$ and $1<p_{\mathrm{T}}^{\text {assoc }}<2 \mathrm{GeV} / c$ as a function of the number of participating nucleons $\left(\mathrm{N}_{\text {part }}\right)$ in $\mathrm{PbPb}$ collisions at $\sqrt{s_{N N}}=$ $2.76 \mathrm{TeV}$.

Following this assumption in Eq. 2, the $v_{\mathrm{n}}^{\mathrm{f}}$ with $1<p_{\mathrm{T}}^{\text {assoc }}<2 \mathrm{GeV} / c$ are extracted as a function of $p_{\mathrm{T}}^{\text {trig }}$ for various centrality classes. The associated particle $p_{\mathrm{T}}^{\text {assoc }}$ range of $1-2 \mathrm{GeV} / \mathrm{c}$ is chosen to minimize the non-flow effects. More details of this procedure can be found in Ref. [15]. Various orders of flow harmonics, $v_{\mathrm{n}}^{\mathrm{f}}$, extracted from the long-range $(2<|\Delta \eta|<4)$ dihadron azimuthal correlations for $1<p_{\mathrm{T}}^{\text {trig }}<2 \mathrm{GeV} / c$ and $1<p_{\mathrm{T}}^{\text {assoc }}<2 \mathrm{GeV} / c$, are shown in Fig. 8 as a function of $\mathrm{N}_{\text {part }}$ in each centrality bin. $v_{2}^{\mathrm{f}}$ shows a strong dependence on the collision centrality, whereas $v_{3}^{\mathrm{f}}$ is only weakly 
centrality dependent for $\mathrm{N}_{\text {part }}>50$. This behavior is consistent with the expectation that $v_{3}^{\mathrm{f}}$ is mainly driven by fluctuations of the initial geometry. Recent theoretical progress has demonstrated that higher order harmonics are more sensitive to the finer structure of the medium (or finite mean-free-path), and thus the shear viscosity [11]. Therefore, measurements of $v_{\mathrm{n}}^{\mathrm{f}}$ up to higher orders provide important input to better constrain the viscous property of the medium, and also shed light on the initial condition of the colliding system.

In summary, the CMS detector at the LHC has been used to measure angular correlations between charged particles in $\Delta \eta$ and $\Delta \phi$ up to $|\Delta \eta| \approx 4$ and over the full range of $\Delta \phi$ in pp collisions at $\sqrt{s}=7 \mathrm{TeV} \mathrm{PbPb}$ collisions at $\sqrt{s_{N N}}=2.76 \mathrm{TeV}$. A longrange ridge-like structure is observed at the near-side $(\Delta \phi \approx 0)$ for the first time in pp collisions. Comprehensive studies of the ridge correlation structure in high multiplicity pp events, as a function of event multiplicity and particle transverse momentum are presented. In $\mathrm{PbPb}$, the extracted 2-D associated yield distributions show a variety of characteristic features that are not present in minimum bias pp interactions. Shortand long-range azimuthal correlations have been studied as a function of the transverse momentum of the trigger particles. The observed long-range ridge-like structure is most evident in the intermediate transverse momentum range, $2<p_{\mathrm{T}}^{\text {trig }}<6 \mathrm{GeV} / c$, and decreases to almost zero for $p_{\mathrm{T}}^{\text {trig }}$ above $10-12 \mathrm{GeV} / c$. A Fourier decomposition of the 1-D $\Delta \phi$-projected correlation functions in the ridge region $(2<|\Delta \eta|<4)$ has been presented. Higher order flow harmonics are extracted as a function of centrality and transverse momentum, providing essential insights to the viscosity and initial condition of the $\mathrm{PbPb}$ collision system. The very broad solid-angle coverage of the CMS detector and the statistical accuracy of the sample analyzed provide significantly improved observations of short- and long-range particle correlations over previously available measurements.

\section{References}

[1] STAR Collaboration, Phys. Rev. Lett. 90 (2003) 082302, arXiv:nucl-ex/0210033.

[2] STAR Collaboration, Phys. Rev. C80 (2009) 064912, arXiv:0909.0191.

[3] PHENIX Collaboration, Phys. Rev. Lett. 98 (2007) 232302, arXiv:nucl-ex/0611019.

[4] PHENIX Collaboration, Phys. Rev. C78 (2008) 014901, arXiv:0801.4545

[5] PHOBOS Collaboration, Phys. Rev. Lett. 104 (2010) 062301, arXiv:0903.2811.

[6] PHOBOS Collaboration, Phys. Rev. C81 (2010) 024904, arXiv:0812.1172.

[7] CMS Collaboration, JHEP 09 (2010) 091, arXiv:1009.4122.

[8] CMS Collaboration, JINST 0803 (2008) S08004.

[9] CMS Collaboration, arXiv:1105.2438.

[10] B. Alver and G. Roland, Phys. Rev. C81 (2010) 054905, arXiv:1003.0194.

[11] B. H. Alver et al., Phys. Rev. C82 (2010) 034913, arXiv:1007.5469.

[12] B. Schenke, S. Jeon, and C. Gale, Phys. Rev. Lett. 106 (2011) 042301, arXiv:1009.3244.

[13] D. Teaney and L. Yan, arXiv:1010.1876.

[14] S. Voloshin and Y. Zhang, Z. Phys. C70 (1996) 665, arXiv:hep-ph/9407282

[15] CMS Collaboration, CMS Physics Analysis Summary HIN-11-006 (2011). 